\title{
A reliable numerical analysis for stochastic dengue epidemic model with incubation period of virus
}

\author{
Ali Raza ${ }^{1 *}$, Muhammad Shoaib Arif ${ }^{1}$ and Muhammad Rafiq ${ }^{2}$
}

${ }^{*}$ Correspondence:

160865@students.au.edu.pk

${ }^{1}$ Department of Mathematics, Air

University, Islamabad, Pakistan

Full list of author information is

available at the end of the article

\section{Springer}

\begin{abstract}
This article represents a numerical analysis for a stochastic dengue epidemic model with incubation period of virus. We discuss the comparison of solutions between the stochastic dengue model and a deterministic dengue model. In this paper, we have shown that the stochastic dengue epidemic model is more realistic as compared to the deterministic dengue epidemic model. The effect of threshold number $R_{1}$ holds in the stochastic dengue epidemic model. If $R_{1}<1$, then situation helps us to control the disease while $R_{1}>1$ shows the persistence of disease in population. Unfortunately, the numerical methods like Euler-Maruyama, stochastic Euler, and stochastic Runge-Kutta do not work for large time step sizes. The proposed framework of stochastic nonstandard finite difference scheme (SNSFD) is independent of step size and preserves all the dynamical properties like positivity, boundedness, and dynamical consistency.
\end{abstract}

Keywords: Dengue virus; Stochastic differential equations; Euler-Maruyama method; Stochastic Euler method; Stochastic Runge-Kutta method; Stochastic NSFD method

\section{Introduction}

Dengue is the most common infection caused by virus transmitted by mosquitos. Dengue is incurable disease. The transmission of dengue virus exists in a cycle. An infected human is bitten by a mosquito which bites another human and the cycle continues. Dengue is found mostly during and after the rainy season in the tropical and sub-tropical areas of the world [1].

In the middle of the twentieth century the disease was restricted geographically. But during the Second World War the eggs of Aedes aegypti were transported by cargo, which played a crucial role in the spreading of the virus over a larger area. Before this, the disease was considered to be relatively small. During the epidemics in Philippines and Thailand, the disease was documented for the first time properly. It is found in more than 100 countries of Asia, America, and Africa. According to a survey of WHO, annually 50 to 100 million infections occur in the world [2]. Dengue fever spreads through a mosquito called Aedes aegypti, and it is a viral infection. The tropical and subtropical countries are the major host of this viral infection. Pakistan is also one of them. Dengue virus has four types [3]. A person who has recovered from one type of dengue virus would gain immu-

(c) The Author(s) 2019. This article is distributed under the terms of the Creative Commons Attribution 4.0 International License (http://creativecommons.org/licenses/by/4.0/), which permits unrestricted use, distribution, and reproduction in any medium, provided you give appropriate credit to the original author(s) and the source, provide a link to the Creative Commons license, and indicate if changes were made. 
nity from the same type for the rest of his life, but he is not protected from the other three types of the virus. World Health Organization disclosed that 50 million dengue infections are present in the world and 2.5 billion are at the risk of getting infection from this virus.

Dengue fever has also ability to convert into dengue hemorrhagic fever (DHF) or dengue shock syndrome (DSS). In DHF the patient suffers from bleeding, low level of blood platelets, and also from plasma leakage; whereas in DSS the blood pressure of the patient drops dangerously. No medicine or vaccine is still discovered to treat dengue fever. The only way to control the disease is to control the source of spreading disease which is Aedes aegypti mosquito. At the beginning this mosquito breeds in the water storage containers, discarded water containers, used automobile tires, and other items which could store rainwater. The only way to control the reproduction of mosquito is proper disposal of rainwater and proper water storage practices [4].

Aedes mosquitoes are said to be a predominant cause of transmission of dengue virus. It has been observed that these mosquitoes commonly reside in the regions with latitudes between $35^{\circ}$ north and $35^{\circ}$ south and an elevation below 1000 meters $(3300 \mathrm{ft})$. Aedes mosquitoes bite their prey approximately two times, i.e.,early in the morning and in the evening. However, there is a possibility that they can bite during other times of the day, and hence infection may spread. World Health Organization (WHO) [2] reports relate that nearly 390 million victims of dengue fever do exist over the world, whereas 96 million call for medical treatment. Every year about 500,000 cases of hemorrhagic fever and the most severe type of dengue disease requires hospitalization. Around $40 \%$ of the world's population resides in dengue endemic areas. According to the WHO estimates, approximately 22,000 persons die every year including a high number of children.

Dengue fever is an old disease. It was found in Chinese region in $610 \mathrm{AD}$ and again in $922 \mathrm{AD}$. The dengue virus multiplied in the eighteenth and nineteenth centuries in many big cities of the world. Dengue plague extended after WW2 (World War Two). It was first isolated in Japan in 1943. In 1944, the virus was isolated at many parts of the world including Indian city Calcutta. Its severe form occurred for the first time in Manila in 1953 to 1954. Dengue expanded in Asia geographically from southeast Asian countries west to India, Sri Lanka, Maldives, and Pakistan. This horrible disease attacked USA in 1980. Similarly, African area was unknown to this disease before 1980. In 1997 dengue virus and Aedes aegypti had a worldwide distribution in tropical and subtropical regions.

Malaysia was also reported to be its victim from 2000 to 2012. A list of 237 identified relevant cases and 28 data sources were found satisfying for the inclusion criteria. A survey was reported between 2000 and 2011 in Thailand to describe the dengue disease dynamics. 610 relevant searches were identified, 40 of which fulfilled the inclusion criteria. The number of dengue cases increased during 2001, 2002, 2008, and 2010. These cases were more fatal in young children. In a survey reported between 2000 and 2010 in Brazil, 51 out of 714 identified relevant searches fulfilled the insertion criteria. In Brazil the epidemiological design of dengue fever disease is complex due to influence of multiple socioenvironmental factors. This work studies a model with incubation periods of dengue virus in human and vector populations. A susceptible person bitten by an infectious vector cannot transfer dengue virus instantly. Such an individual is called an infected human during this period. Intrinsic incubation period of dengue virus in human is about five days [5]. 
When an infectious person is bitten by the susceptible vector, then prior to its infectious state it will be an infected vector. On the other hand, the extrinsic incubation period of dengue virus in vector population is about ten days [5]. An uninfected person with no immunity to resist the dengue virus is called a susceptible person. A victim of dengue infection who reacquires their immunity is named a recovered person.

Mathematical modelling has emerged as an effective tool to extract comprehensive insight about epidemic diseases. The formation of the model and the possible simulations allow for analyzing the sensitivity and comparison of conjuncture paradigms. As a result, the prediction of mediator, host, and ecological factor affecting public health is possible, and health policy makers can scientifically suggest and implement health services [2]. Several studies have been conducted on various models of dengue transmission dynamics [6]. It is well known that nonlinear initial value problems (IVPs) do not always possess analytical solutions. The available classical explicit finite-difference schemes, such as RungeKutta and Euler methods, can bring about misleading chaos and deceitful oscillations for certain intensities of the discretization parameters [7-11]. Due to these reasons and some other scheme-dependent numerical instabilities such methods prove to be less privileged options.

In general, the determination of stochastic differential equations (SDEs) is either difficult, or these stochastic differential equations (SDEs) do not have the explicit solutions. We use different numerical schemes to integrate these equations [12-15]. A natural question on numerical schemes can be the following despite the convergence analysis: Do the numerical schemes preserve the dynamical properties of the initial system [16]?

In the deterministic modeling we have observed that the usual numerical schemes like Euler and Runge-Kutta do not preserve dynamical properties. Even in the stochastic case the Euler-Maruyama, stochastic Euler, and atochastic Runge-Kutta do not preserve the dynamical properties. This is the question: Can we construct a stochastic numerical scheme which preserves all the dynamical properties?

The main theme of this paper is to introduce the notion of stochastic nonstandard finite difference scheme (SNSFD) based on the rules introduced in the deterministic case by Mickens [17-19].

The strategy of this paper is as follows:

In Sect. 2, we remind classical definitions and some history of stochastic differential equations (SDEs). In Sect. 3, we explain the formulation of stochastic epidemic models. In Sect. 4, we discuss the deterministic dengue model and equilibrium points. In Sect. 5, we explore the stochastic dengue model. In Sect. 6, we introduce different stochastic numerical schemes and compare their result with deterministic solutions. In Sect. 7, we draw the conclusion and give the future directions.

\section{Preliminaries}

Einstein is considered the pioneer of the idea of stochastic differential equations (SDEs) as he gave a mathematical assembly between microscopic random motion of particles and the macroscopic diffusion equation in 1905 [20]. Nowadays the SDEs attract a lot of attention due to physical developments in real life systems because the ordinary differential equations (ODEs) did not apprehend random forcing and stochastic inputs. A stochas- 
tic calculus delivers a mathematical substance for the conduct of stochastic differential equations (SDEs). In general form, we can write the stochastic differential equation that comprises parameters, a continuous time $t$ and variable $X_{t}$, as follows:

$$
d X_{t}=u\left(t, X_{t}\right) d t+v\left(t, X_{t}\right) d B(t)
$$

and the integral form is

$$
X(t)=c+\int_{t_{o}}^{t} u\left(s, X_{s}\right) d s+\int_{t_{o}}^{t} v\left(s, X_{s}\right) d B_{s} .
$$

The above stochastic differential equation is also called Ito's stochastic differential equation (SDE) where $u\left(t, X_{t}\right)$ and $v\left(t, X_{t}\right)$ are drift and diffusion coefficients, respectively. The random variable $c$ is called the initial value at instant $t_{0}$. A solution $X_{t}$ of Eq. (1) or (2) is called stochastic process.

\subsection{Brownian motion}

The Brownian motion can be defined as a continuous time random walk with the following properties [21]:

(i) $B_{0}=0$.

(ii) $B_{t}$ must be continuous, the event happens with probability one. The sample trajectories $t \rightarrow B_{t}$ are continuous with probability one.

(iii) For any finite sequence of times $t_{1}<t_{2}<t_{3}<\cdots<t_{n}$, the following paths $B_{t_{1}}-B_{t_{o}}$, $B_{t_{2}}-B_{t_{1}}, B_{t_{3}}-B_{t_{2}}, \ldots, B_{t_{n}}-B_{t_{n-1}}$ are independent.

(iv) For any times $0 \leq s \leq t, B_{t}-B_{s}$ is normally distributed with mean zero and variance is $t-s$. In particularly, we say that

$$
\text { Expectation }\left[B_{t}-B_{s}\right]=0 \text { and Variance }\left[B_{t}-B_{s}\right]=t-s \text {. }
$$

The stochastic processes are a fundamental example of Brownian motion. A stochastic epidemic model is articulated in tenure of stochastic modeling process. A stochastic modeling process is an assemblage of random variables $\left\{Y_{t}(S) / t \in T, s \in S\right\}$, where $T$ is the guide set and $S$ is a joint sample space. The guide set often characterizes time such as $T=\{0,1,2, \ldots\}$ or $T=[0, \infty)$. Time may be discrete or continuous. The homework of stochastic modeling procedures is founded on probability theory. The stochastic epidemic modeling processes can be described in three ways: DTMC (discrete time Markov chain) epidemic models, CTMC (continuous time Markov chain) epidemic models, and SDEs (stochastic differential equations) epidemic models. In discrete time Markov chain (DTMC) epidemic models, we assumed that time and the state variables are discrete. In continuous time Markov chain (CTMC) epidemic models, the time is continuous and the state variables are discrete. In stochastic differential equations (SDEs) epidemic models, we presumed that both the time and the state variables are continuous. Now we discuss stochastic differential equations (SDEs) of epidemic models. They were first introduced and developed by Itó (1942). Stochastic processes evolved almost in all sciences such as economics, mathematics, physics, chemistry, and biology. The solutions of stochastic differential equations (SDEs) are not given explicitly due to their non-differentiable character 
of realization of the Brownian motion. So, the stochastic numerical approximation is used to study the properties of stochastic epidemic models [22].

\section{Formulation of stochastic epidemic models}

Epidemics are generally fashioned by nonlinear systems observed through fragmentary noisy data. This modeling can be divided into two main classes as deterministic epidemic models and stochastic epidemic models. It is interesting to note that after the deterministic system the ordinary differential equations have been articulated for the population dynamics. We can derive several different classes of stochastic epidemic models that consider the irregular nature of the population processes in a persistent manner. The idea here is to apprehend unknown impacts such as changing behaviors, public interventions, seasonal effects, and social cycles. There are various ways to transmit the deterministic epidemic models to stochastic epidemic models. This modeling can be done by discrete time Markov chain (DTMC), continuous time Markov chain (CTMC), and Ito's stochastic differential equations (SDEs). Therefore, the Ito stochastic differential equations give strong arguments for moving away from deterministic epidemic models to stochastic epidemic models. There are various ways to devise stochastic differential equations such as parametric perturbation and non-parametric perturbation methods. In the parametric perturbation method, a parameter from model is chosen and transformed into random variables. In the non-parametric perturbation method, Brownian processes are added to each differential equation (or extra stochasticity parameters are introduced). This type of modeling is more useful as compared to the parametric perturbation method. Another way of the non-parametric perturbation method was introduced by Allen [23-28] in which any extra stochasticity parameters are not introduced in the model. Here we formulate the ways of the non-parametric perturbation method in epidemic models and use different numerical methods to prompt them and check the efficiency of numerical methods on stochastic epidemic models. We observe the relationship between the solutions of deterministic epidemic models and stochastic epidemic models.

\section{Deterministic dengue model}

In this segment we introduce the deterministic dengue model with incubation period of virus [1]. At any arbitrary time $t$, the variables are stated as follows: $\overline{S_{h}}(t)$ represents susceptible humans, $\overline{X_{h}}(t)$ represents infected humans, $\overline{I_{h}}(t)$ represents infectious humans, and $\overline{R_{h}}(t)$ represents recovered humans, whereas $\overline{S_{v}}(t)$ represents susceptible mosquitos, $\overline{X_{v}}(t)$ represents infected mosquitos, and $\overline{I_{v}}(t)$ represents infectious mosquitos. The communication dynamics of dengue model is shown in Fig. 1.

The model parameters are described as follows: $N_{T}$ (denotes the total human residents size), $N_{v}$ (denotes the total vector residents size), $\mu_{h}$ (denotes the death rate of human residents), $\beta_{h}$ (denotes the infectious proportion from vector to human residents), $\alpha_{h}$ (denotes the proportion from infected human residents to infectious human residents), $r$ (denotes the immune rate of human residents), $\beta_{v}$ (denotes the proportion of dengue virus from human residents to vector residents), $\mu_{v}$ (denotes the death rate of vector residents), $\alpha_{v}$ (denotes the proportion of infected vector residents to infectious vector residents), and $c$ (denotes the constant recruitment proportion of vector residents). 

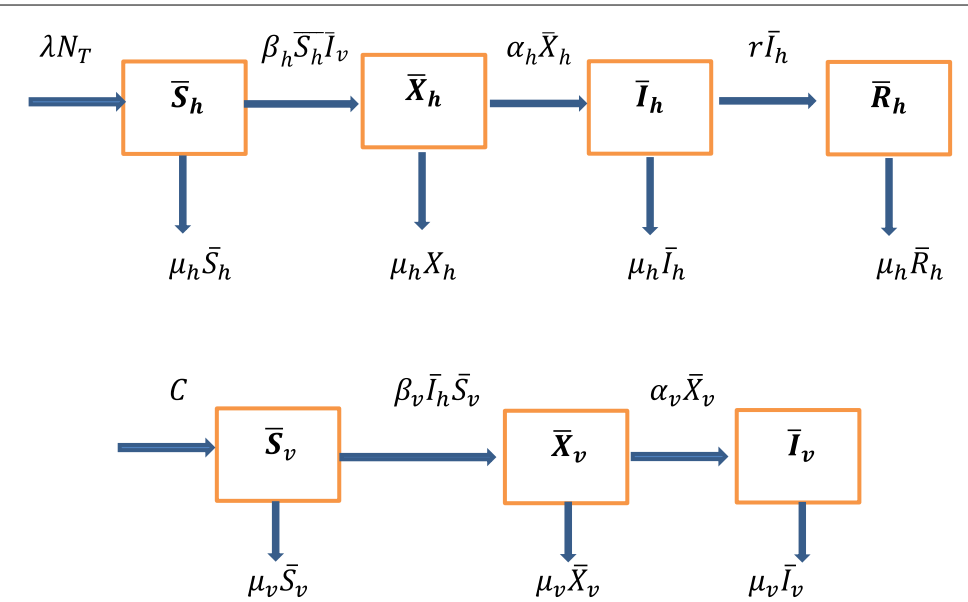

Figure 1 Flow diagram of dengue model

The governing equations of the dengue model are given below:

$$
\begin{aligned}
& \frac{d \overline{S_{h}}}{d t}=\lambda N_{T}-\beta_{h} \overline{S_{h} I_{v}}-\mu_{h} \overline{S_{h}} \\
& \frac{d \overline{X_{h}}}{d t}=\beta_{h} \overline{S_{h} I_{v}}-\left(\alpha_{h}+\mu_{h}\right) \overline{X_{h}} \\
& \frac{d \overline{I_{h}}}{d t}=\alpha_{h} \overline{X_{h}}-\left(r+\mu_{h}\right) \overline{I_{h}} \\
& \frac{d \overline{R_{h}}}{d t}=r \overline{I_{h}}-\mu_{h} \overline{R_{h}} \\
& \frac{d \overline{S_{v}}}{d t}=c-\beta_{v} \overline{I_{h} S_{v}}-\mu_{v} \overline{S_{v}} \\
& \frac{d \overline{X_{v}}}{d t}=\beta_{v} \overline{I_{h} S_{v}}-\left(\alpha_{v}+\mu_{v}\right) \overline{X_{v}} \\
& \frac{d \overline{I_{v}}}{d t}=\alpha_{v} \overline{X_{v}}-\mu_{v} \overline{I_{v}}
\end{aligned}
$$

where the constant size of total human and vector residents respectively is as follows:

$$
\begin{aligned}
& N_{T}=\overline{S_{h}}+\overline{X_{h}}+\overline{I_{h}}+\overline{R_{h}}, \\
& N_{v}=\overline{S_{v}}+\overline{X_{v}}+\overline{I_{v}} .
\end{aligned}
$$

Therefore,

$$
\frac{d N_{T}}{d t}=0 \Rightarrow \lambda=\mu_{h} \quad \text { and } \quad \frac{d N_{v}}{d t}=0 \Rightarrow N_{v}=\frac{c}{\mu_{v}}
$$

The normalized form of dengue model (3) is

$$
\begin{aligned}
& \frac{d S}{d t}=\mu_{h}-\beta_{h} S I_{v}\left(\frac{c}{\mu_{v}}\right)-\mu_{h} S \\
& \frac{d X}{d t}=\beta_{h} S I_{v}\left(\frac{c}{\mu_{v}}\right)-\alpha_{h} X-\mu_{h} X \\
& \frac{d I}{d t}=\alpha_{h} X-r I-\mu_{h} I, \\
& \frac{d X_{v}}{d t}=\beta_{v} I N_{T}\left(1-X_{v}-I_{v}\right)-\alpha_{v} X_{v}-\mu_{v} X_{v}, \\
& \frac{d I_{v}}{d t}=\alpha_{v} X_{v}-\mu_{v} I_{v}
\end{aligned}
$$


where $S=\frac{\overline{S_{h}}}{N_{T}}, X=\frac{\overline{X_{h}}}{N_{T}}, I=\frac{\overline{I_{h}}}{N_{T}}, X_{v}=\frac{\overline{X_{v}}}{N_{v}}, I_{v}=\frac{\overline{I_{v}}}{N_{v}}$ with condition $S+X+I=1$,

$$
S_{v}+X_{v}+I_{v}=1
$$

\subsection{Steady state of the dengue model}

The steady state of dengue model (6) can be categorized into two ways of equilibrium points as follows:

Disease-free equilibrium is $D_{1}=(1,0,0,0,0)$.

Endemic equilibrium is $E_{1}=\left(S^{o}, X^{o}, I^{o}, X_{v}^{o}, I_{v}^{o}\right)$.

$$
\begin{aligned}
& S^{o}=\frac{\left(\alpha_{v}+\mu_{v}\right)\left(M N \mu_{\lambda}^{2} \mu_{v}+\alpha_{v} \gamma_{v} \mu_{h}\right)}{\alpha_{h} \gamma_{v}\left(\mu_{h}\left(\alpha_{v}+\mu_{v}\right)+\alpha_{v} \gamma_{h}\right)}, \\
& X^{o}=\frac{M \mu_{\lambda}^{2} \mu_{v}\left(\alpha_{v}+\mu_{v}\right)\left(R_{1}-1\right)}{\alpha_{h} \alpha_{h}\left(\mu_{h}\left(\alpha_{v}+\mu_{v}\right)+\alpha_{v} \gamma_{h}\right)}, \\
& I^{o}=\frac{\mu_{h} \mu_{v}\left(\alpha_{v}+\mu_{v}\right)\left(R_{1}-1\right)}{\alpha_{h}\left(\mu_{h}\left(\alpha_{v}+\mu_{v}\right)+\alpha_{v} \gamma_{h}\right)} \\
& X_{v}^{o}=\frac{\mu_{v}\left(M N \mu_{h}^{3} \mu_{v}\right)\left(R_{1}-1\right)}{\alpha_{v} \gamma_{h}\left(\alpha_{h} \mu_{h} \gamma_{v}+M N \mu_{h}^{2} \mu_{v}\right)}, \\
& I_{v}^{o}=\frac{\left(M N \mu_{h}^{3} \mu_{v}\right)\left(R_{1}-1\right)}{\gamma_{h}\left(\alpha_{h} \mu_{h} \gamma_{v}+M N \mu_{h}^{2} \mu_{v}\right)},
\end{aligned}
$$

where

$$
\begin{aligned}
& R_{1}=\frac{\alpha_{h} \alpha_{v} \gamma_{v} \gamma_{h}}{\mu_{v}\left(r+\mu_{h}\right)\left(\alpha_{v}+\mu_{v}\right)\left(\alpha_{h}+\mu_{h}\right)}, \quad M=\frac{r+\mu_{h}}{\mu_{h}}, \\
& N=\frac{\alpha_{h}+\mu_{h}}{\mu_{h}}, \quad \gamma_{h}=\frac{\beta_{h} C}{\mu_{v}}, \quad \gamma_{v}=\beta_{v} N_{T} .
\end{aligned}
$$

Note that $R_{1}$ is the reproductive number of dengue model (6). The reproductive number has a vital role in disease dynamics. If the reproductive number $R_{1}<1$, then this strategy helps us to control the disease, and if $R_{1}>1$, then the alarming situation of disease in population occurs. If $R_{1}=1$, then the disease will start diffusion in residents.

\section{Stochastic dengue model}

Let $Y=\left[S, X, I, X_{v}, I_{v}\right]^{T}$ to form the stochastic differential equations (SDEs) of model (6). We need to compute the expectations $E^{*}[\Delta Y]$ and $E^{*}\left[\Delta Y \Delta Y^{T}\right]$. To find these expectations, the possible changes along with their associated transition probabilities are listed in Table 1.

The expectation and variance of dengue model (6) is defined as

$$
\begin{aligned}
& E^{*}[\Delta Y]=\sum_{i=1}^{n} P_{i}(\Delta Y)_{i}, \\
& \text { Expectation }=E^{*}[\Delta Y]=\left[\begin{array}{c}
\mu_{h}-\beta_{h} S I_{v}\left(\frac{c}{\mu_{v}}\right)-\mu_{h} S \\
\beta_{h} S I_{v}\left(\frac{c}{\mu_{v}}\right)-\alpha_{h} X-\mu_{h} X \\
\alpha_{h} X-r I-\mu_{h} I \\
\beta_{v} I N_{T}\left(1-X_{v}-I_{v}\right)-\alpha_{v} X_{v}-\mu_{v} X_{v} \\
\alpha_{v} X_{v}-\mu_{v} I_{v}
\end{array}\right] \Delta t,
\end{aligned}
$$


Table 1 Transition probabilities

\begin{tabular}{ll}
\hline Transition & Probabilities \\
\hline$(\Delta Y)_{1}=[1,0,0,0,0]^{T}$ & $P_{1}=\mu_{h} \Delta t$ \\
$(\Delta Y)_{2}=[-1,1,0,0,0]^{T}$ & $P_{2}=\beta_{h} S_{v}\left(\frac{c}{\mu_{v}}\right) \Delta t$ \\
$(\Delta Y)_{3}=[-1,0,0,0,0]^{T}$ & $P_{3}=\mu_{h} S \Delta t$ \\
$(\Delta Y)_{4}=[0,-1,1,0,0]^{T}$ & $P_{4}=\alpha_{h} X \Delta t$ \\
$(\Delta Y)_{5}=[0,-1,0,0,0]^{T}$ & $P_{5}=\mu_{h} X \Delta t$ \\
$(\Delta Y)_{6}=[0,0,-1,0,0]^{T}$ & $P_{6}=\left(r+\mu_{h}\right) \Delta t$ \\
$(\Delta Y)_{7}=[0,0,0,1,0]^{T}$ & $P_{7}=\beta_{v} / N_{T} \Delta t$ \\
$(\Delta Y)_{8}=[0,0,0,-1,0]^{T}$ & $P_{8}=\beta_{v} / N_{T} X_{v} \Delta t$ \\
$(\Delta Y)_{9}=[0,0,0,-1,0]^{T}$ & $P_{9}=\beta_{v} / N_{T} I_{v} \Delta t$ \\
$(\Delta Y)_{10}=[0,0,0,-1,1]^{T}$ & $P_{10}=\alpha_{v} X_{v} \Delta t$ \\
$(\Delta Y)_{11}=[0,0,0,-1,0]^{T}$ & $P_{11}=\mu_{v} X_{v} \Delta t$ \\
$(\Delta y)_{12}=[0,0,0,0,-1]^{T}$ & $P_{12}=\mu_{v} I_{v} \Delta t$ \\
\hline &
\end{tabular}

$\operatorname{Var}=E^{*}\left[\Delta Y \Delta Y^{T}\right]=\sum_{i=1}^{n} P_{i}\left[(\Delta Y)_{i}\right]\left[(\Delta Y)_{i}\right]^{T}$,

$E^{*}\left[\Delta Y \Delta Y^{T}\right]$

$=\left[\begin{array}{ccccc}\mu_{h}+\beta_{h} S I_{\nu}\left(\frac{c}{\mu_{v}}\right)+\mu_{h} S & -\beta_{h} S I_{v}\left(\frac{c}{\mu_{v}}\right) & 0 & 0 & 0 \\ -\beta_{h} S I_{\nu}\left(\frac{c}{\mu_{v}}\right) & \beta_{h} S I_{v}\left(\frac{c}{\mu_{v}}\right)+\alpha_{h} X+\mu_{h} X & -\alpha_{h} X & 0 & 0 \\ 0 & -\alpha_{h} X & r I+\mu_{h} I & 0 & 0 \\ 0 & 0 & 0 & \beta_{v} I N_{T}\left(1+X_{v}+I_{v}\right)+\alpha_{v} X_{v}+\mu_{v} X_{v} & 0 \\ 0 & 0 & 0 & 0 & \mu_{v} I_{v}\end{array}\right] \Delta t$.

The stochastic differential equation satisfies the diffusion processes. So,

$$
\frac{d Y(t)}{d t}=f(Y(t), t)+L(Y(t), t) \frac{d B(t)}{d t} .
$$

If we define

$$
\text { drift }=f(Y(t), t)=\frac{E^{*}[\Delta Y]}{\Delta t}
$$

and

$$
\text { diffusion }=L(Y(t), t)=\sqrt{\frac{E^{*}\left[\Delta Y \Delta Y^{T}\right]}{\Delta t}},
$$

then the stochastic differential equation of dengue model (6) is

$$
d Y(t)=f(Y(t), t) d t+L(Y(t), t) d B(t)
$$

with initial conditions $Y(0)=Y_{o}=[0.1,0.0001,0.0001,0.001,0.001]^{T}, 0 \leq t \leq T$, and $B(t)$ is the Brownian motion.

\subsection{Euler-Maruyama method}

Here we use the Euler-Maruyama method [29] to find the numerical solution of SDEs (7) of the dengue model by using the parameter values given in [1] (see Table 2).

We can write the Euler-Maruyama method of SDEs (7) as

$$
Y_{n+1}=Y_{n}+f\left(Y_{n}, t\right) \Delta t+L\left(Y_{n}, t\right) d B(t)
$$


Table 2 Parameter values [1]

\begin{tabular}{lll}
\hline Parameters & Values (Days) & \\
\cline { 2 - 3 } & DFE & EE \\
\hline$\mu_{h}$ & 0.0000391 & 0.0000391 \\
$\beta_{h}$ & 0.00005 & 0.00005 \\
$\alpha_{h}$ & $1 / 5$ & $1 / 5$ \\
$r$ & $1 / 14$ & $1 / 14$ \\
$N T$ & 5000 & 5000 \\
$\beta_{V}$ & 0.00008 & 0.00008 \\
$\mu_{V}$ & $1 / 14$ & $1 / 14$ \\
$\alpha_{V}$ & $1 / 10$ & $1 / 10$ \\
$c$ & 3 & 300 \\
$\sigma_{1}$ & 0.0004 & 0.0004 \\
$\sigma_{2}$ & 0.0003 & 0.0003 \\
$\sigma_{3}$ & 0.0002 & 0.0002 \\
$\sigma_{4}$ & 0.0001 & 0.0001 \\
$\sigma_{5}$ & 0.0001 & 0.0001 \\
\hline
\end{tabular}

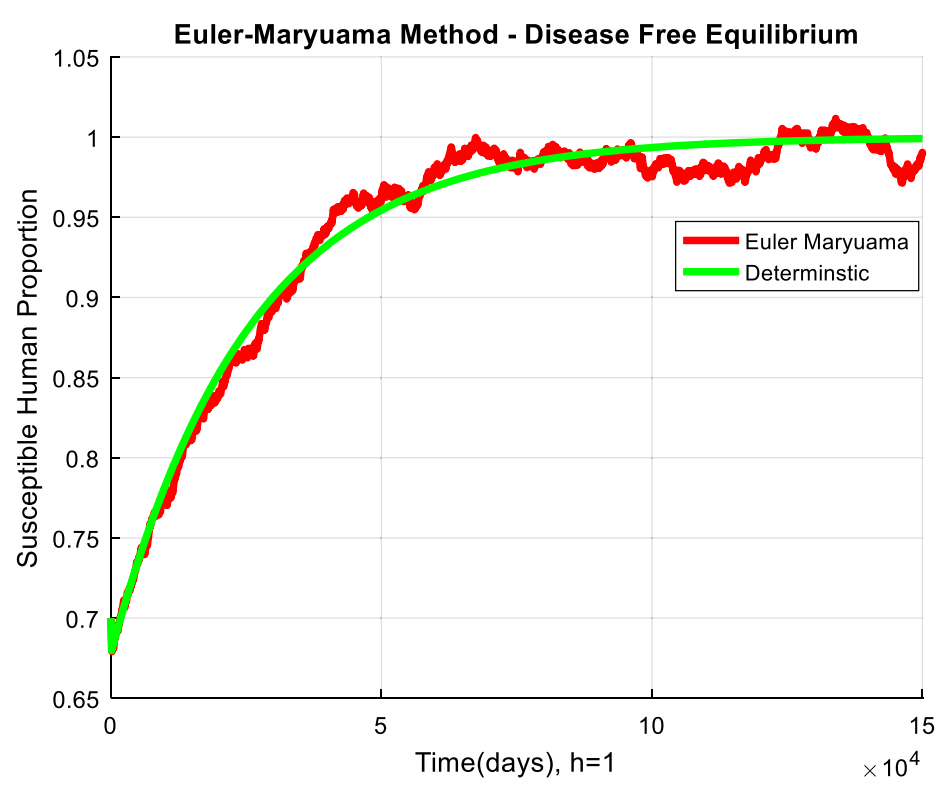

Figure 2 In susceptible human proportion Euler-Maruyama converges to disease-free equilibrium while deterministic solution is the mean of Euler-Maruyama solution for $h=1$

where ' $\Delta t$ ' is time step size. The solution of SDEs lies in confidence interval for both disease-free equilibrium and endemic equilibrium as shown in Figs. 2 and 3. The solution of deterministic dengue model for the disease-free equilibrium $D_{1}=(1,0,0,0,0)$ and the reproductive number $R_{1}=0.0960<1$ means these measures help to control the disease in residents. The endemic equilibrium $E_{1}=(0.1042,0.0001751,0.00049,0.001144,0.001601)$ and the reproductive number $R_{1}=9.5969>1$ means disease is endemic in residents. We have observed here that the solutions of SDEs are most probabilistic as compared to the solutions of deterministic ODEs and also the solutions of deterministic ODEs are the mean of solutions of SDEs. 


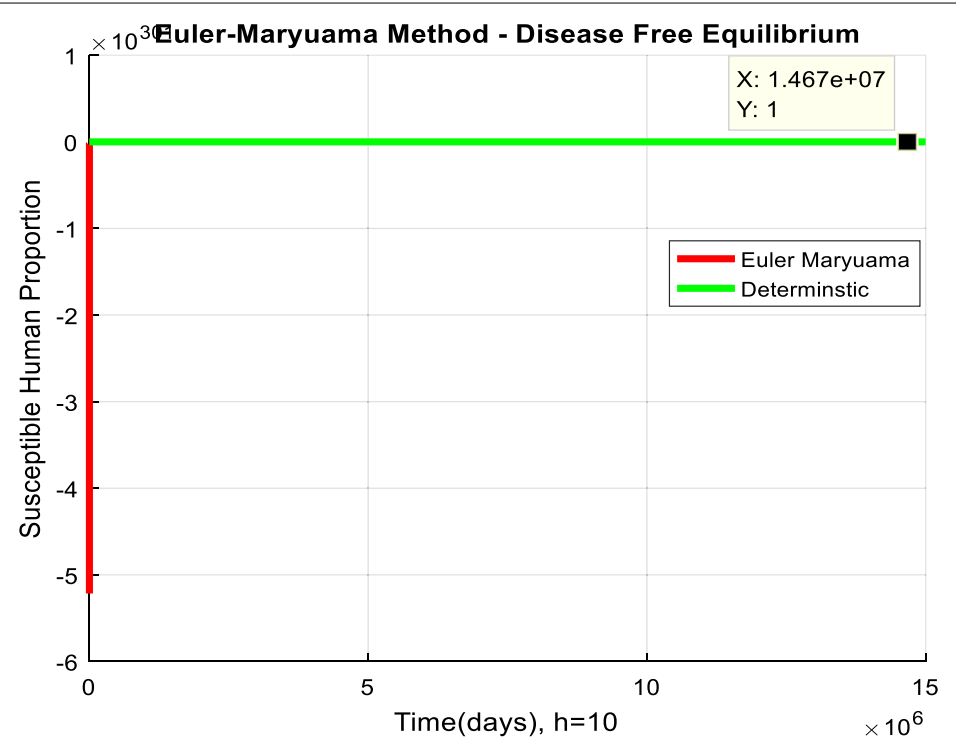

Figure 3 In susceptible human proportion Euler-Maruyama shows negativity and divergence for disease-free equilbrium for $h=10$

\section{Non-parametric perturbation stochastic dengue model}

Another way to construct the SDEs from the deterministic ODEs is to introduce the non-parametric perturbation in each differential equation of dengue model (6) as follows:

$$
\begin{aligned}
& d S=\left(\mu_{h}-\beta_{h} S I_{v}\left(\frac{c}{\mu_{v}}\right)-\mu_{h} S\right) d t+\sigma_{1} d B_{1}(t) S, \\
& d X=\left(\beta_{h} S_{\nu}\left(\frac{c}{\mu_{v}}\right)-\alpha_{h} X-\mu_{h} X\right) d t+\sigma_{2} d B_{2}(t) X, \\
& d I=\left(\alpha_{h} X-r I-\mu_{h} I\right) d t+\sigma_{3} d B_{3}(t) I, \\
& d X_{v}=\left(\beta_{v} I N_{T}\left(1-X_{v}-I_{v}\right)-\alpha_{v} X_{v}-\mu_{v} X_{v}\right) d t+\sigma_{4} d B_{4}(t) X_{v}, \\
& d I_{v}=\left(\alpha_{v} X_{v}-\mu_{v} I_{v}\right) d t+\sigma_{5} d B_{5}(t) I_{v},
\end{aligned}
$$

where $\sigma_{1}, \sigma_{2}, \sigma_{3}, \sigma_{4}$, and $\sigma_{5}$ are stochasticity of each compartment of the dengue model and $B_{j}(t),(j=1,2,3,4,5)$ are the independent Brownian motions. The non-parametric perturbation stochastic dengue model does not have the explicit solutions due to nondifferentiability term of Brownian motion, so we introduce some new stochastic numerical methods to find the solution of model (8).

\subsection{Stochastic Euler method}

The stochastic Euler method can be derived from the above non-parametric perturbation stochastic dengue model (8) as follows:

$$
\begin{aligned}
& S^{n+1}=S^{n}+h\left[\mu_{h}-\beta_{h} S^{n} I_{v}^{n}\left(\frac{c}{\mu_{v}}\right)-\mu_{h} S^{n}+\sigma_{1} d B_{1}(t) S^{n}\right], \\
& X^{n+1}=X^{n}+h\left[\beta_{h} S^{n} I_{v}^{n}\left(\frac{c}{\mu_{v}}\right)-\alpha_{h} X^{n}-\mu_{h} X^{n}+\sigma_{2} d B_{2}(t) X^{n}\right], \\
& I^{n+1}=I^{n}+h\left[\alpha_{h} X^{n}-r I^{n}-\mu_{h} I^{n}+\sigma_{3} d B_{3}(t) I^{n}\right], \\
& X_{v}^{n+1}=X_{v}^{n}+h\left[\beta_{v} I^{n} N_{T}\left(1-X_{v}^{n}-I_{v}^{n}\right)-\alpha_{v} X_{v}^{n}-\mu_{v} X_{v}^{n}+\sigma_{4} d B_{4}(t) X_{v}^{n}\right], \\
& I_{v}^{n+1}=I_{v}^{n}+h\left[\alpha_{v} X_{v}^{n}-\mu_{v} I_{v}^{n}+\sigma_{5} d B_{5}(t) I_{v}^{n}\right],
\end{aligned}
$$


where $h$ is any time step size, and we simulate the solution of stochastic Euler method by using MATLAB program and parameter values given in [1] (see Table 2).

\subsection{Stochastic Runge-Kutta method}

The stochastic Runge-Kutta method can be derived from the above non-parametric perturbation stochastic dengue model (8) as follows:

$$
\begin{aligned}
& A_{1}=h\left[\mu_{h}-\beta_{h} S^{n} I_{v}^{n}\left(\frac{c}{\mu_{v}}\right)-\mu_{h} S^{n}+\sigma_{1} d B_{1}(t) S^{n}\right], \\
& B_{1}=h\left[\beta_{h} S^{n} I_{v}^{n}\left(\frac{c}{\mu_{v}}\right)-\alpha_{h} X^{n}-\mu_{h} X^{n}+\sigma_{2} d B_{2}(t) X^{n}\right] \text {, } \\
& C_{1}=h\left[\alpha_{h} X^{n}-r I^{n}-\mu_{h} I^{n}+\sigma_{3} d B_{3}(t) I^{n}\right] \text {, } \\
& D_{1}=h\left[\beta_{v} I^{n} N_{T}\left(1-X_{v}^{n}-I_{v}^{n}\right)-\alpha_{v} X_{v}^{n}-\mu_{v} X_{v}^{n}+\sigma_{4} d B_{4}(t) X_{v}^{n}\right] \text {, } \\
& E_{1}=h\left[\alpha_{v} X_{v}^{n}-\mu_{v} I_{v}^{n}+\sigma_{5} d B_{5}(t) I_{v}^{n}\right. \text {, } \\
& A_{2}=h\left[\mu_{h}-\beta_{h}\left(S^{n}+\frac{A_{1}}{2}\right)\left(I_{v}^{n}+\frac{E_{1}}{2}\right)\left(\frac{c}{\mu_{v}}\right)-\mu_{h}\left(S^{n}+\frac{A_{1}}{2}\right)+\sigma_{1} d B_{1}(t)\left(S^{n}+\frac{A_{1}}{2}\right)\right] \text {, } \\
& B_{2}=h\left[\beta_{h}\left(S^{n}+\frac{A_{1}}{2}\right)\left(I_{v}^{n}+\frac{E_{1}}{2}\right)\left(\frac{c}{\mu_{v}}\right)-\alpha_{h}\left(X^{n}+\frac{B_{1}}{2}\right)\right. \\
& \left.-\mu_{h}\left(X^{n}+\frac{B_{1}}{2}\right)+\sigma_{2} d B_{2}(t)\left(X^{n}+\frac{B_{1}}{2}\right)\right], \\
& C_{2}=h\left[\alpha_{h}\left(X^{n}+\frac{B_{1}}{2}\right)-r\left(I^{n}+\frac{C_{1}}{2}\right)-\mu_{h}\left(I^{n}+\frac{C_{1}}{2}\right)+\sigma_{3} d B_{3}(t)\left(I^{n}+\frac{C_{1}}{2}\right)\right], \\
& D_{2}=h\left[\beta_{v}\left(I^{n}+\frac{C_{1}}{2}\right) N_{T}\left(1-\left(X_{v}^{n}+\frac{D_{1}}{2}\right)-\left(I_{v}^{n}+\frac{E_{1}}{2}\right)\right)-\alpha_{v}\left(X_{v}^{n}+\frac{D_{1}}{2}\right)\right. \\
& \left.-\mu_{v}\left(X_{v}^{n}+\frac{D_{1}}{2}\right)+\sigma_{4} d B_{4}(t)\left(X_{v}^{n}+\frac{D_{1}}{2}\right)\right] \text {, } \\
& E_{2}=h\left[\alpha_{v}\left(X_{v}^{n}+\frac{D_{1}}{2}\right)-\mu_{v}\left(I_{v}^{n}+\frac{E_{1}}{2}\right)+\sigma_{5} d B_{5}(t)\left(I_{v}^{n}+\frac{E_{1}}{2}\right)\right] \text {, } \\
& A_{3}=h\left[\mu_{h}-\beta_{h}\left(S^{n}+\frac{A_{2}}{2}\right)\left(I_{v}^{n}+\frac{E_{2}}{2}\right)\left(\frac{c}{\mu_{v}}\right)-\mu_{h}\left(S^{n}+\frac{A_{2}}{2}\right)+\sigma_{1} d B_{1}(t)\left(S^{n}+\frac{A_{2}}{2}\right)\right] \text {, } \\
& B_{3}=h\left[\beta_{h}\left(S^{n}+\frac{A_{2}}{2}\right)\left(I_{v}^{n}+\frac{E_{2}}{2}\right)\left(\frac{c}{\mu_{v}}\right)-\alpha_{h}\left(X^{n}+\frac{B_{2}}{2}\right)\right. \\
& \left.-\mu_{h}\left(X^{n}+\frac{B_{2}}{2}\right)+\sigma_{2} d B_{2}(t)\left(X^{n}+\frac{B_{2}}{2}\right)\right], \\
& C_{3}=h\left[\alpha_{h}\left(X^{n}+\frac{B_{2}}{2}\right)-r\left(I^{n}+\frac{C_{2}}{2}\right)-\mu_{h}\left(I^{n}+\frac{C_{2}}{2}\right)+\sigma_{3} d B_{3}(t)\left(I^{n}+\frac{C_{2}}{2}\right)\right], \\
& D_{3}=h\left[\beta_{v}\left(I^{n}+\frac{C_{2}}{2}\right) N_{T}\left(1-\left(X_{v}^{n}+\frac{D_{2}}{2}\right)-\left(I_{v}^{n}+\frac{E_{2}}{2}\right)\right)-\alpha_{v}\left(X_{v}^{n}+\frac{D_{2}}{2}\right)\right. \\
& \left.-\mu_{v}\left(X_{v}^{n}+\frac{D_{2}}{2}\right)+\sigma_{4} d B_{4}(t)\left(X_{v}^{n}+\frac{D_{2}}{2}\right)\right] \text {, } \\
& E_{3}=h\left[\alpha_{v}\left(X_{v}^{n}+\frac{D_{2}}{2}\right)-\mu_{v}\left(I_{v}^{n}+\frac{E_{2}}{2}\right)+\sigma_{5} d B_{5}(t)\left(I_{v}^{n}+\frac{E_{2}}{2}\right)\right] \text {, } \\
& A_{4}=h\left[\mu_{h}-\beta_{h}\left(S^{n}+A_{3}\right)\left(I_{v}^{n}+E_{3}\right)\left(\frac{c}{\mu_{v}}\right)-\mu_{h}\left(S^{n}+A_{3}\right)+\sigma_{1} d B_{1}(t)\left(S^{n}+A_{3}\right)\right] \text {, } \\
& B_{4}=h\left[\beta_{h}\left(S^{n}+A_{3}\right)\left(I_{v}^{n}+E_{3}\right)\left(\frac{c}{\mu_{v}}\right)-\alpha_{h}\left(X^{n}+B_{3}\right)\right. \\
& \left.-\mu_{h}\left(X^{n}+B_{3}\right)+\sigma_{2} d B_{2}(t)\left(X^{n}+B_{3}\right)\right] \\
& C_{4}=h\left[\alpha_{h}\left(X^{n}+B_{3}\right)-r\left(I^{n}+C_{3}\right)-\mu_{h}\left(I^{n}+C_{3}\right)+\sigma_{3} d B_{3}(t)\left(I^{n}+C_{3}\right)\right], \\
& D_{4}=h\left[\beta_{v}\left(I^{n}+C_{3}\right) N_{T}\left(1-\left(X_{v}^{n}+D_{3}\right)-\left(I_{v}^{n}+E_{3}\right)\right)-\alpha_{v}\left(X_{v}^{n}+D_{3}\right)\right. \\
& \left.-\mu_{v}\left(X_{v}^{n}+D_{3}\right)+\sigma_{4} d B_{4}(t)\left(X_{v}^{n}+D_{3}\right)\right] \text {, } \\
& E_{4}=h\left[\alpha_{v}\left(X_{v}^{n}+D_{3}\right)-\mu_{v}\left(I_{v}^{n}+E_{3}\right)+\sigma_{5} d B_{5}(t)\left(I_{v}^{n}+E_{3}\right)\right] \text {, } \\
& S^{n+1}=S^{n}+\frac{1}{6}\left[A_{1}+2 A_{2}+2 A_{3}+A_{4}\right] \text {, } \\
& X^{n+1}=X^{n}+\frac{1}{6}\left[B_{1}+2 B_{2}+2 B_{3}+B_{4}\right] \\
& I^{n+1}=I^{n}+\frac{1}{6}\left[C_{1}+2 C_{2}+2 C_{3}+C_{4}\right] \text {, } \\
& X_{v}^{n+1}=X_{v}^{n}+\frac{1}{6}\left[D_{1}+2 D_{2}+2 D_{3}+D_{4}\right] \text {, } \\
& I_{v}^{n+1}=I_{v}^{n}+\frac{1}{6}\left[E_{1}+2 E_{2}+2 E_{3}+E_{4}\right] \text {, }
\end{aligned}
$$

where $h$ is any time step size, and we simulate the solution of stochastic RungeKutta method by using MATLAB program and parameter values given in [1] (see Table 2).

\subsection{Stochastic NSFD method}

The proposed framework of stochastic nonstandard finite difference method (SNSFD) can be derived from the above non-parametric perturbation stochastic dengue model (8) as 
follows:

$$
\begin{aligned}
& S^{n+1}=\frac{S^{n}+\phi(h)\left[\mu_{h}+\sigma_{1} d B_{1}(t) S^{n}\right]}{\left(1+\phi(h) \beta_{h} h_{\nu}\left(\frac{c}{\mu_{\nu}}\right)+\phi(h) \mu_{h}\right)}, \\
& X^{n+1}=\frac{X^{n}+\phi(h)\left[\beta_{h} S^{n} I_{\nu}^{n}\left(\frac{c}{\mu_{\nu}}\right)+\sigma_{2} d B_{2}(t) X^{n}\right]}{\left(1+\phi(h) \alpha_{h}+\phi(h) \mu_{h}\right)}, \\
& I^{n+1}=\frac{I^{n}+\phi(h)\left[\alpha_{h} X^{n}+\sigma_{3} d B_{3}(t) I^{n}\right]}{\left(1+\phi(h) r+\phi(h) \mu_{h}\right)}, \\
& X_{v}^{n+1}=\frac{X_{v}^{n}+\phi(h)\left[\beta_{v} I^{n} N_{T}\left(1+I_{v}^{n}\right)+\sigma_{4} d B_{4}(t) X_{v}^{n}\right]}{\left(1+\phi(h) \beta_{v} I^{n} N_{T}+\phi(h) \alpha_{v}+h \mu_{v}\right)} \text {, } \\
& I_{v}^{n+1}=\frac{I_{v}^{n}+\phi(h)\left[\alpha_{v} X_{v}^{n}+\sigma_{5} d B_{5}(t) I_{v}^{n}\right]}{\left(1+\phi(h) \mu_{v}\right)} \text {, }
\end{aligned}
$$

where $\phi(h)=1-\mathrm{e}^{-h}$ and " $h$ " is any time step size. We simulate the solution of the proposed framework of stochastic nonstandard finite difference (SNSFD) method by using MATLAB program and parameter values given in [1] (see Table 2).

\section{Results and discussion}

In Fig. 2, we observe that the Euler-Maruyama method converges to steady states of the dengue disease model, while deterministic solution is the mean of Euler-Maruyama solution for discretization parameter $h=1$. When we increase the time step size, we see in Fig. 3 that the Euler-Maryuama method fails to maintain positivity and shows divergence for disease-free equilbrium. Consequently, the Euler-Maryuama method does not work for certain time step sizes. In Figs. 4 and 6, we observe that the stochastic Euler method converges to equilibrium point, while deterministic solution is the mean of stochastic Euler solution for $h=1$. When we increase the time step size, we see in Figs. 5 and 7 that the stochastic Euler method fails to maintain positivity and consistency for both disease-free and endemic equilbria. So the stochastic Euler method is not a reliable technique to find the solutions of stochastic dengue disease model. In Figs. 8 and 10, we observe that the stochastic Runge-Kutta method converges to disease-free equilbrium and endemic equi-

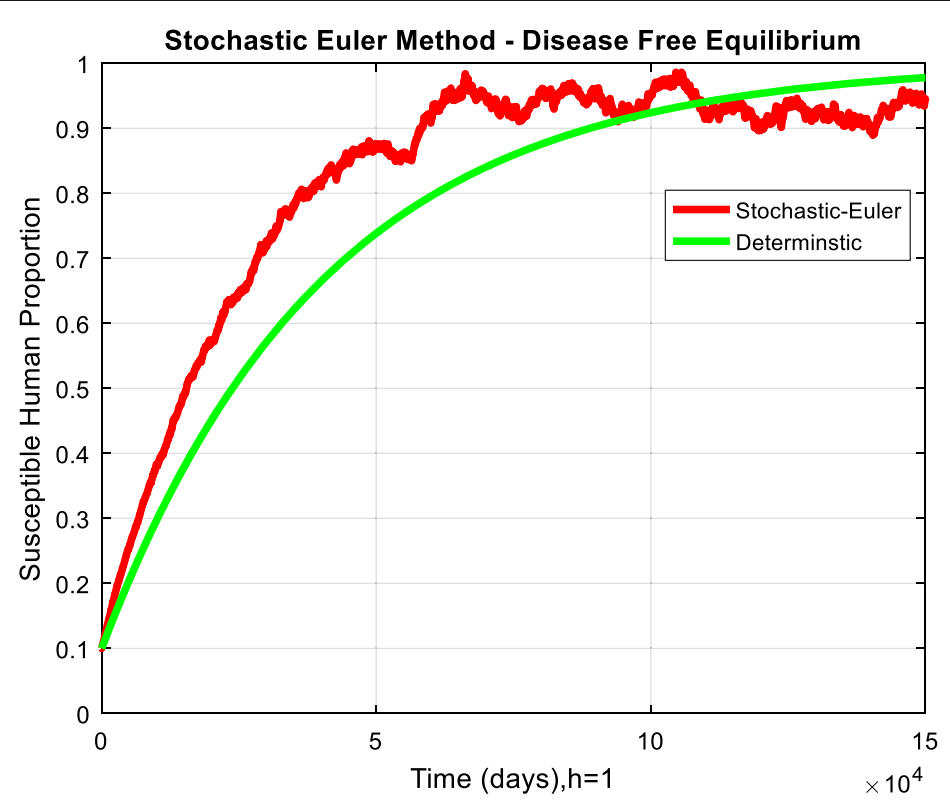

Figure 4 In susceptible human proportion stochastic Euler method converges to disease-free equilibrium while deterministic solution is the mean of stochastic Euler method solution for $h=1$ 


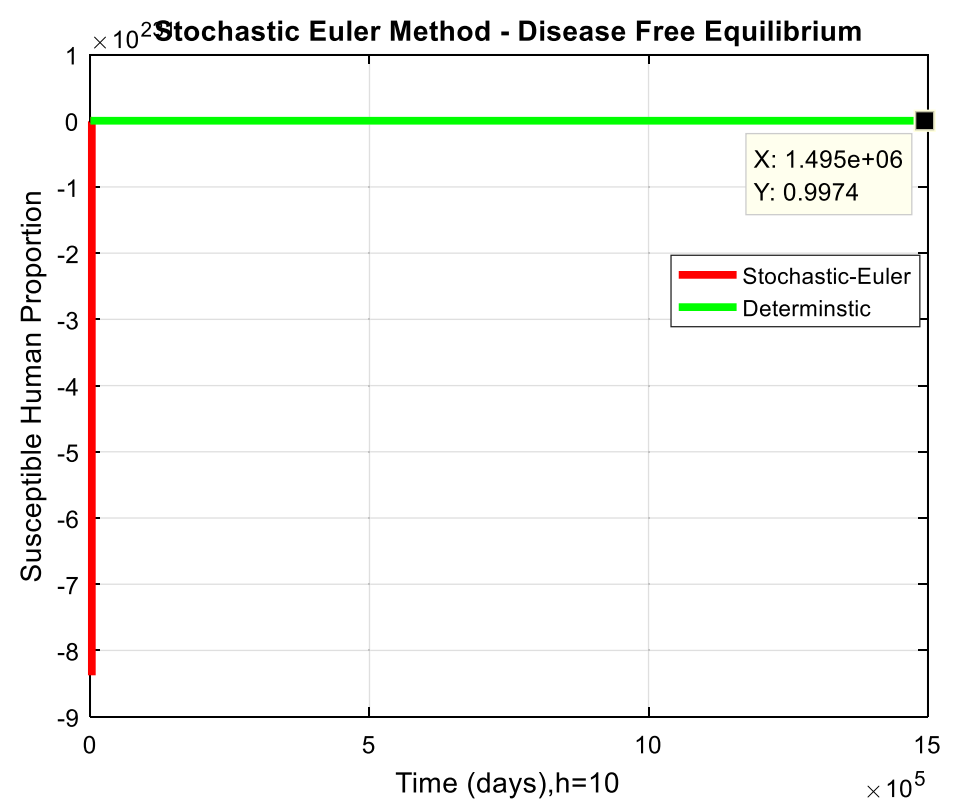

Figure $\mathbf{5}$ In susceptible human proportion stochastic Euler method fails to maintain the positivity and diverges for disease-free equilbrium for $h=10$

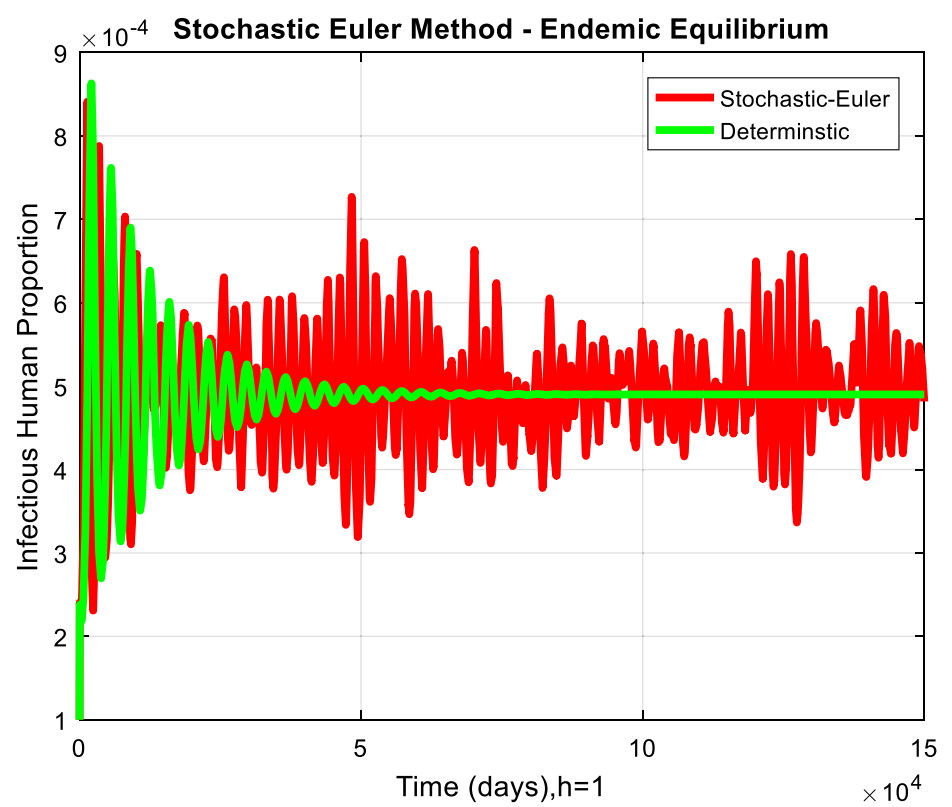

Figure 6 In infectious human proportion stochastic Euler method converges to endemic equilibrium while deterministic solution is the mean of stochastic Euler method solution for $h=1$

lbrium, while deterministic solution is the mean of stochastic Runge-Kutta solution for step size $h=1$. When we increase the time step size, we see in Figs. 9 and 11, the stochastic Runge-Kutta method fails to maintain stability and positivity for both disease-free and endemic equilbrium points. Thus the aforasaid stochastic schemes do not preserve all the dynamical properities [15, 21, 23]. In Figs. 12 to 15, we observe that the stochastic NSFD 


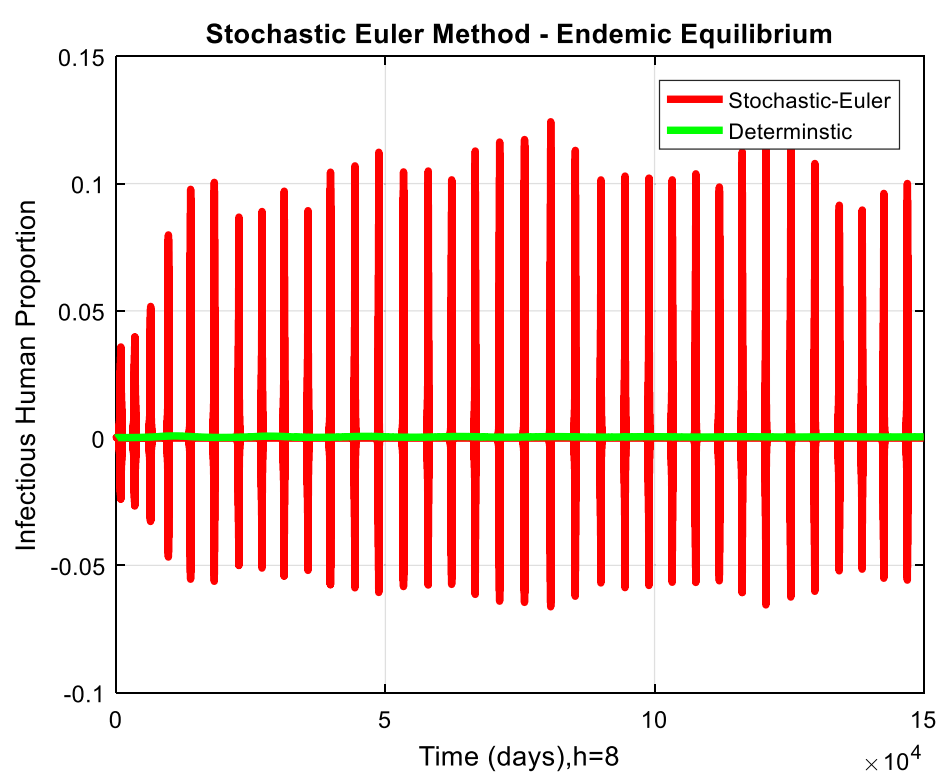

Figure 7 In infectious human proportion stochastic Euler method shows negativity and unstable behavior for endemic equilibrium for $h=8$

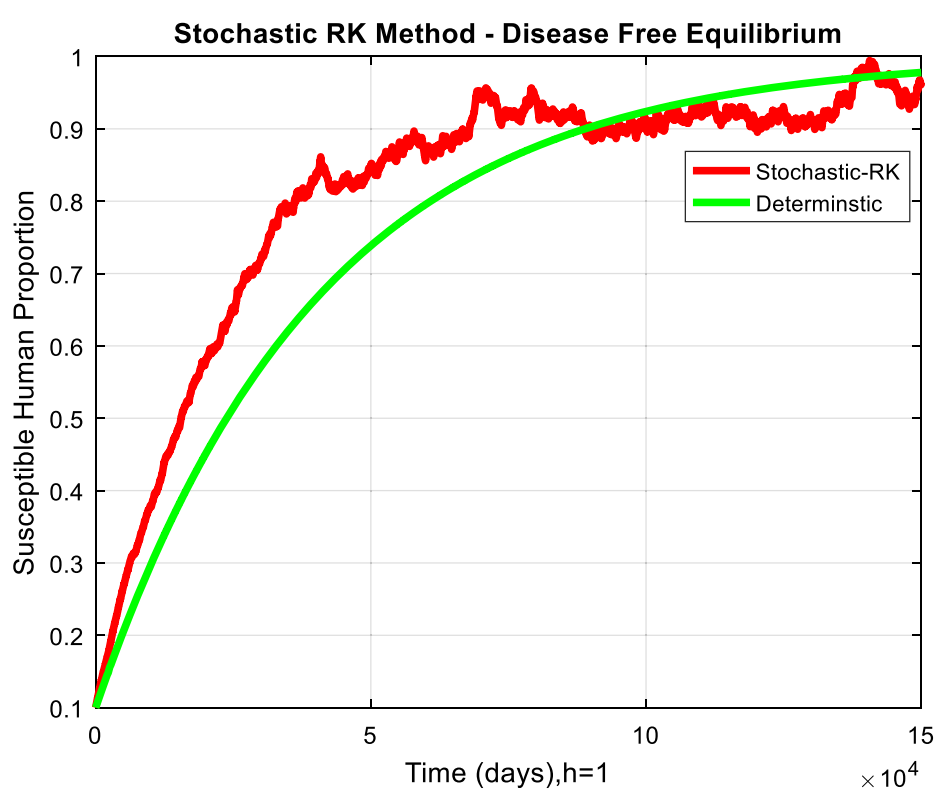

Figure 8 In suscepitable human proportion stochastic Runge-Kutta method converges to disease-free equilbrium while deterministic solution is the mean of stochastic Runge-Kutta method solution for $h=1$

method converges for both disease-free and endemic equilbrium points, while deterministic solution is the mean of stochastic NSFD solution for any discretization such as $h=1$ and $h=100$. So the stochastic NSFD method preserves all the dynamical properties such as positivity, boundedness, and dynamical consistency defined by Mickens in the stochastic context. The proposed stochastic NSFD scheme is independent of step size and remains convergent for any time step size. 


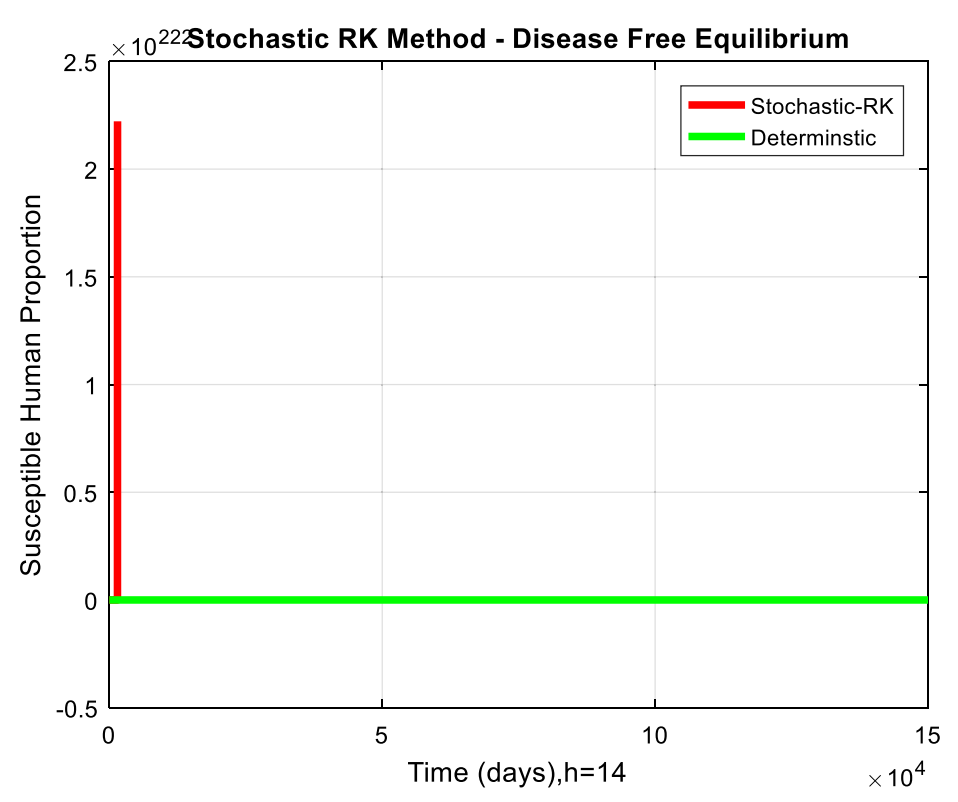

Figure 9 In suscepitable human proportion stochastic Runge-Kutta method solution is unbound for disease-free equilibrium for $h=14$

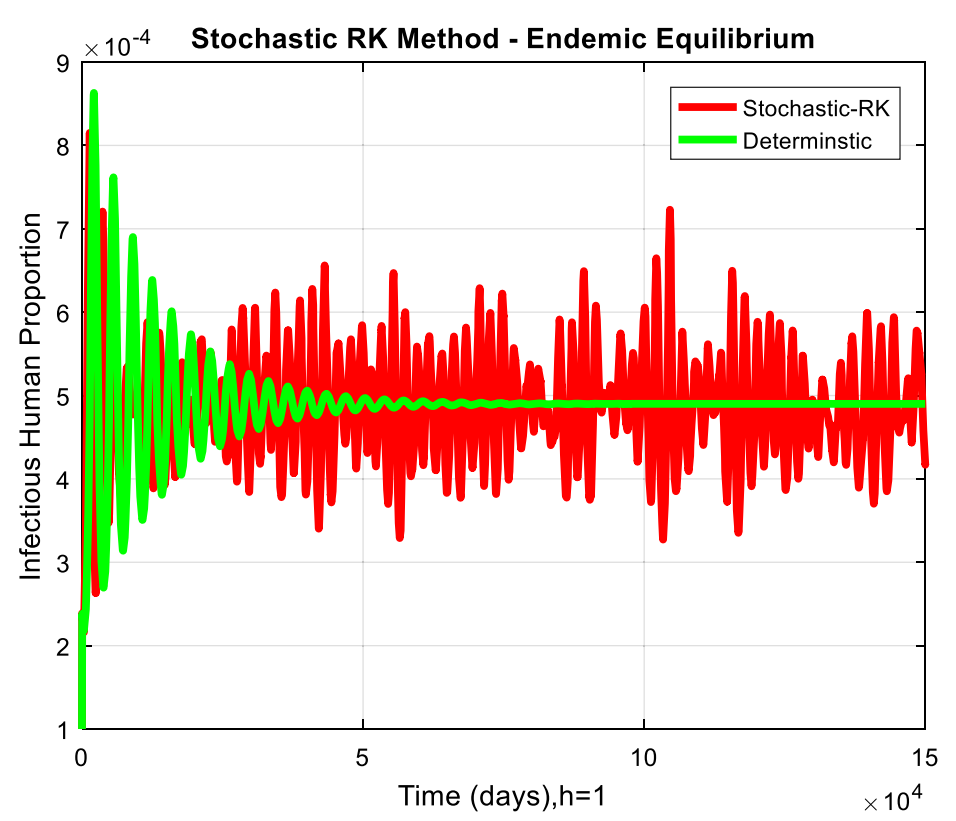

Figure 10 In infectious human proportion stochastic Runge-Kutta method converges to endemic equilibrium while deterministic solution is the mean of stochastic Runge-Kutta method solution for $h=1$

\section{Conclusion and future framework}

The stochastic dengue epidemic model is a more suitable strategy as compared to the deterministic epidemic model to understand the dengue disease dynamics with incubation period of virus. The Euler-Maruyama method, stochastic Euler method, stochastic Runge-Kutta method converge to the true equilibrium points for very small time step 


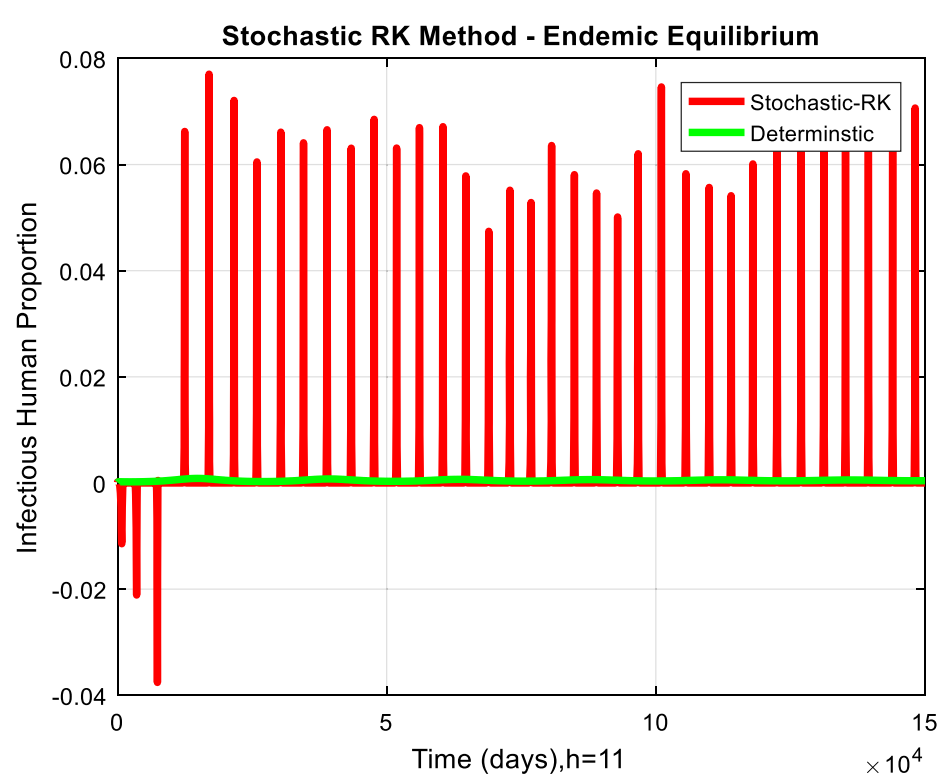

Figure 11 In infectious human proportion stochastic Runge-Kutta method shows failure of dynamical properties for endemic equilibrium for $h=11$

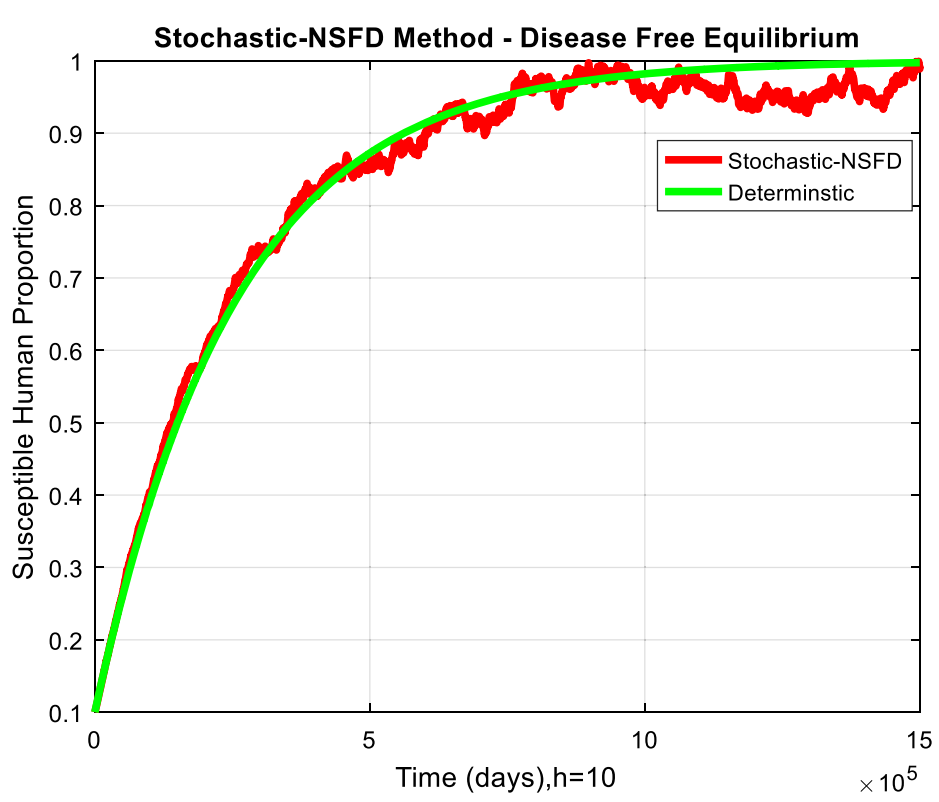

Figure 12 In suscepitable human proportion stochastic-NSFD converges to disease-free equilibrium while deterministic solution is the mean of stochastic NSFD solution for $h=1$

sizes. After increasing the time step size, these methods diverge and lose the dynamical properties such as positivity, bounded-ness, and dynamical consistency. The proposed framework of stochastic nonstandard finite difference method (SNSFD) of dengue model works for any time step size. The proposed (SNSFD) scheme is suitable for all types of nonlinear and complicated epidemic models. The stochastic solutions are very close with the deterministic solutions. The study of stochastic epidemic models plays the most im- 


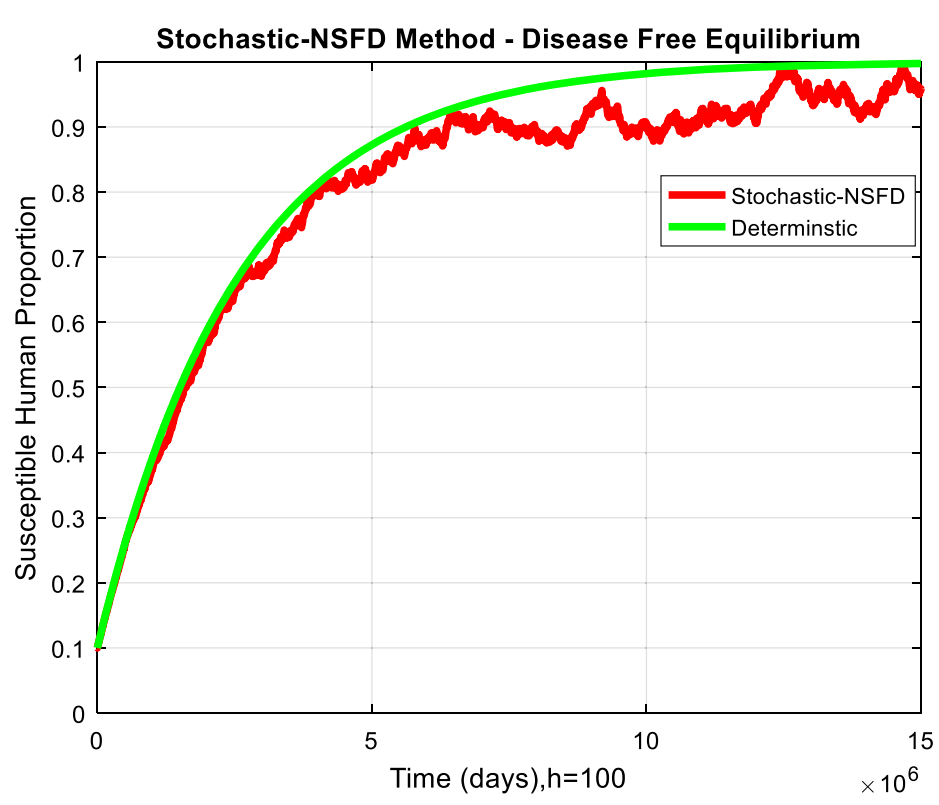

Figure 13 In suscepitable human proportion stochastic-NSFD preserve all dynamical properties for disease-free equilibrium for $h=100$

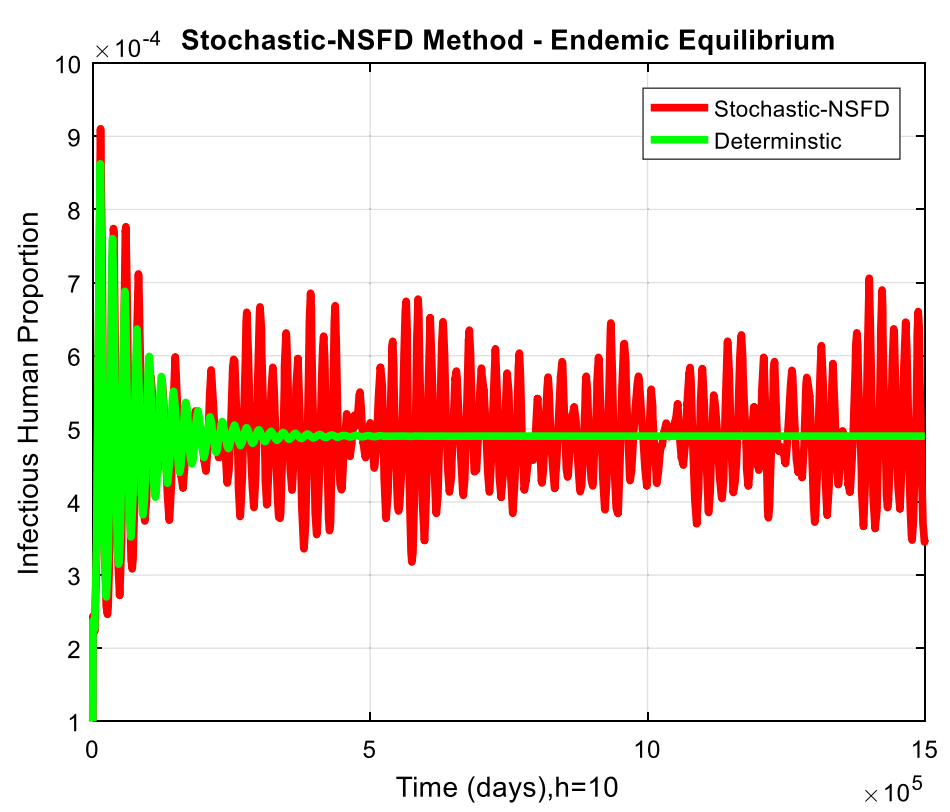

Figure 14 In infectious human proportion stochastic-NSFD converges to endemic equilibrium while deterministic solution is the mean of stochastic NSFD solution for $h=10$

portant role in disease dynamics. We have observed that stochastic epidemic models are more realistic as compared to deterministic epidemic models. As the future work, the proposed (SNSFD) scheme could be implemented to stochastic delay epidemic models and stochastic diffusion epidemic models. The numerical analysis proposed in this work could also be extended to fractional order dynamical systems [30, 31]. Our future plan also in- 


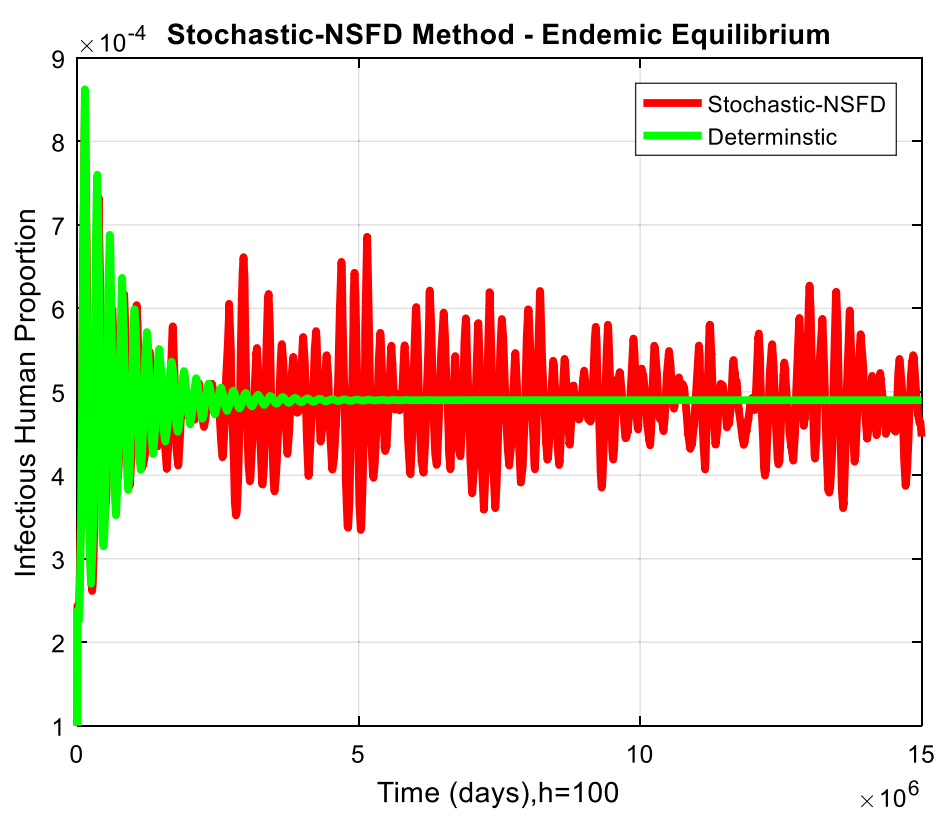

Figure 15 In infectious human proportion stochastic-NSFD preserve all dynamical properties for endemic equilibrium while deterministic solution is the mean of stochastic NSFD solution for $h=100$

cludes the construction of reliable numerical schemes for the solutions of fractional order stochastic epidemic models for various infectious diseases.

Acknowledgements

We would like to thank the referees for their valuable comments.

\section{Funding}

No funding is available for this research project.

\section{Abbreviations}

CTMC, Continuous Time Markov Chain; DTMC, Discrete Time Markov Chain; DHF, Dengue Hemorrhagic Fever; DSS, Dengue Shock Syndrome; DFE, Disease-Free Equilibrium; EE, Endemic Equilibrium; NSFD, Nonstandard Finite Difference; ODEs, Ordinary Differential Equations; SDE, Stochastic Differential Equation; SNSFD, Stochastic Nonstandard Finite Difference; WHO, World Health Organization; WW2, World War 2.

\section{Competing interests}

The authors declare that they have no competing interests.

\section{Authors' contributions}

The authors have achieved equal contributions. All authors read and approved the manuscript.

\section{Author details}

${ }^{1}$ Department of Mathematics, Air University, Islamabad, Pakistan. ${ }^{2}$ Faculty of Engineering, University of Central Punjab, Lahore, Pakistan.

\section{Publisher's Note}

Springer Nature remains neutral with regard to jurisdictional claims in published maps and institutional affiliations.

Received: 7 June 2018 Accepted: 15 January 2019 Published online: 28 January 2019

\section{References}

1. Pongsumpun, P.: Mathematical model of dengue disease with incubation period of virus. Int. J. Math. Comput. Sci., 2 , 8 (2008)

2. World Health Organization: Dengue Hemorrhagic Fever, Diagnosis Treatment and Control. World Health Organization, Geneva (1997) 
3. Laureano-Rosario, A.E., Garcia-Rejon, J.E., Gomez-Carro, S., Farfan-Ale, J.A., Muller-Karger, F.E.: Application of artificial neural networks for dengue fever outbreak predictions in the northwest coast of Yucatan, Mexico and San Juan, Puerto Rico. Trop. Med. Infect. Dis. 3(1), 5 (2018)

4. Laureano-Rosario, A.E., Garcia-Rejon, J.E., Gomez-Carro, S., Farfan-Ale, J.A., Muller-Karger, F.E.: Modelling dengue fever risk in the state of Yucatan, Mexico using regional-scale satellite-derived sea surface temperature. Acta Trop. 172, 50-57 (2017)

5. Guzman, M.G., Kouri, G.: Dengue an update. Lancet Infect. Dis. 2(1), 33-42 (2002)

6. Naowarat, S., Rajabhat, S.: Dynamical model for determining human susceptibility to dengue fever. Am. J. Appl. Sci. 8(11), 1101-1106 (2011)

7. Zafar, Z., Rehan, K., Mushtaq, M.: Fractional-order scheme for bovine babesiosis disease and tick populations. Adv. Differ. Equ. 2017, Article ID 86 (2017)

8. Zafar, Z., Rehan, K., Mushtaq, M., Rafiq, M.: Numerical treatment for nonlinear Brusselator chemical model. J. Differ. Equ. Appl. 23(3), 521-538 (2017)

9. Zafar, Z., Rehan, K., Mushtaq, M., Rafiq, M.: Numerical modeling for nonlinear biochemical reaction networks. Iran. J. Math. Chem. 8(4), 413-423 (2017)

10. Zafar, Z., Ahmad, M.O., Pervaiz, A., Rafiq, M.: Fourth order compact method for one dimensional inhomogeneous telegraph equations with $\mathrm{O}\left(\mathrm{h}^{4}, \mathrm{k}^{3}\right)$. Pak. J. Eng. Appl. Sci. 14, 96-101 (2014)

11. Zafar, Z., Rehan, K., Mushtaq, M.: HIV/AIDS epidemic fractional-order model. J. Differ. Equ. Appl. 23(7), 1298-1315 (2017)

12. Kloeden, P.E.: Numerical Solution of SDE Through Computer Experiments, vol. 1. Springer, Berlin (1994)

13. Kloeden, P.E., Platen, E.: Numerical Solution of Stochastic Differential Equations, vol. 23. Springer, Berlin (1992)

14. Oksendal, B.: Stochastic Differential Equations. Springer, Berlin (2003)

15. Platen, E.: An introduction to numerical methods for stochastic differential equations. Acta Numer. 8, 197-246 (1999)

16. Cresson, J., Pierret, F.: Nonstandard finite difference scheme preserving dynamical properties. arXiv Preprint (2014). arXiv:1410.6661

17. Mickens, R.E.: Nonstandard Finite Difference Models of Differential Equations. World Scientific, River Edge (1994)

18. Mickens, R.E.: Advances in Applications of Nonstandard Finite Difference Schemes. World Scientific, Hackensack (2005)

19. Mickens, R.E.: A fundamental principle for constructing nonstandard finite difference schemes for differential equations. J. Differ. Equ. Appl. 11(7), 645-653 (2005)

20. Gard, T.C.: Introduction to Stochastic Differential Equations. Dekker, New York (1988)

21. Karatzas, I., Shreve, S.E.: Brownian Motion and Stochastic Calculus, 2nd edn. Springer, Berlin (1991)

22. Allen, L.J.S., Burgin, A.: Comparison of deterministic and stochastic SIS and SIR models in discrete time. Math. Biosci. $163,1-33(2000)$

23. Allen, E.J.: Modeling with Ito Stochastic Differential Equations. Springer, Dordrecht (2007)

24. Britton, T.: Stochastic epidemic models. J. Math. Biosci. 225(1), 24-35 (2010)

25. Allen, E.J., Allen, L.J.S., Arciniega, A., Greenwood, P.E.: Construction of equivalent stochastic differential equation models. Stoch. Anal. Appl. 26(2), 274-297 (2008)

26. Keeling, M..., Rohani, P.: Modeling Infectious Diseases in Humans and Animals. Princeton University Press, Princeton (2008)

27. Shoji, I., Ozaki, T.: Comparative study of estimation methods for continuous time stochastic processes. J. Time Ser Anal. 18(5), 485-506 (1997)

28. Bayram, M., Partal, T., Buyukoz, G.O.: Numerical methods for simulation of stochastic differential equations. Adv. Differ. Equ. 2018, 17 (2018)

29. Maruyama, G.: Continuous Markov processes and stochastic equations. Rend. Circ. Mat. Palermo 4(1), $48-90$ (1955)

30. Jajarmi, A., Baleanu, D.: A new fractional analysis on the interaction of HIV with CD4+ T-cells. Chaos Solitons Fractals 113, 221-229 (2018)

31. Baleanu, D., Jajarmi, A., Bonyah, E., Hajipour, M.: New aspects of poor nutrition in the life cycle within the fractional calculus. Adv. Differ. Equ. 2018(1), 230 (2018)

\section{Submit your manuscript to a SpringerOpen ${ }^{\circ}$ journal and benefit from:}

- Convenient online submission

- Rigorous peer review

- Open access: articles freely available online

- High visibility within the field

- Retaining the copyright to your article

Submit your next manuscript at $\gg$ springeropen.com 